\title{
A Huge Aneurysm of the Ascending Aorta in a Young Male with Marfan Syndrome: "Giant Aorta-Great Heart"
}

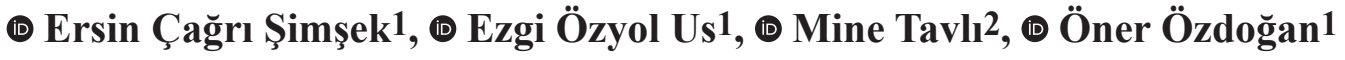

${ }^{1}$ University of Health Sciences, İzmir Tepecik Training and Research Hospital, Clinic of Cardiology, İzmir, Turkey

2University of Health Sciences, İzmir Tepecik Training and Research Hospital, Clinic of Cardiovascular Surgery, İzmir, Turkey

\begin{abstract}
Marfan syndrome, described in the $19^{\text {th }}$ century, is a connective tissue disease inherited as an autosomal dominant, owing to mutations in the FBN1 gene encoding fibrillin-1. Marfan syndrome involves the cardiovascular, ocular, and skeletal systems. Although Marfan syndrome is inherited as an autosomal dominant with high penetrance, about $30 \%$ of cases are due to sporadic mutation. A 34-yearold man with no prior medical history presented with worsening exercise dyspnea. On physical examination, he was tall stature, he had myopia, pectus deformity, pes planus, the wrist and thumb signs. His cardiac examination revealed a grade 3/6 early diastolic murmur over the aortic area. Transthoracic echocardiography revealed a severely dilated left ventricle (left ventricular end diastolic diameter and volume; $9.0 \mathrm{~cm}, 520 \mathrm{~mL}$ respectively), a giant ascending aorta measuring $11.0 \mathrm{~cm}$ and reduced
\end{abstract}

systolic function (left ventricular ejection fraction 50\%). A computed tomography angiography imaging which was ordered to exclude aortic dissection and assess the extent of aortic dilation showed an aortic diameter of $11.6 \times 11 \mathrm{~cm}$ at the widest point without any dissection flap. The patient was diagnosed as having Marfan syndrome according to the Ghent criteria and underwent Benthall procedure. In the literature, huge aneurysm of the ascending aorta without dissection is extremely rare, and the current case with a giant aneurysm of approximately $12 \mathrm{~cm}$ has been treated uneventfully. In conclusion, such huge aortic aneurysms could be asymptomatic and silent for many years until severe aortic regurgitation and aortic dissection occurs.

Keywords: Ascending aorta, giant aneurysm, aortic dilatation, Marfan syndrome, genetics

\footnotetext{
Address for Correspondence: Ersin Çağrı Şimşek, University of Health Sciences, İzmir Tepecik Training and Research Hospital, Clinic of Cardiology, İzmir, Turkey

Phone: +90 23246969 69-3666 e-mail: ercagsim@hotmail.com ORCID ID: orcid.org/0000-0001-6084-0982

Received: 30.05.2018 Accepted: 02.03.2019
}

Cite this article as: Şimşek EÇ, Özyol Us E, Tavlı M, Özdoğan Ö. A Huge Aneurysm of the Ascending Aorta in a Young Male with Marfan Syndrome: “Giant Aorta-Great Heart”. EJCM 2019;7(1):43-46.

DOI: 10.32596/ejcm.galenos.2019.00032 


\section{Introduction}

Marfan syndrome (MFS), described in the $19^{\text {th }}$ century, is a connective tissue disease inherited as an autosomal dominant, owing to mutations in the FBN1 gene encoding fibrillin $1^{(1)}$. MFS involves the cardiovascular, ocular, and skeletal systems; therefore, it is characterized by those three clinical criteria; thoracic aortic aneurysm and/ or dissection, ectopia lentis, and systemic features (with score $\geq 7)^{(2)}$.

In this report, we described a new diagnosed MFS case which has an overly dilated heart owing to severe aortic insufficiency with huge aortic aneurysm

\section{Case Report}

A 34-year-old man with no prior medical history presented with worsening exercise dyspnea and marked limitation in activity due to symptoms for the previous three weeks. Among his family members, nobody had an aortic disease and connective tissue disease. A month prior to the admission he was easily climbing up the fifth floor. On physical examination, he was $184 \mathrm{~cm}$ tall, weighed $79 \mathrm{~kg}$, and he had myopia, pectus deformity, pes planus, and elongated limbs (arm span $194 \mathrm{~cm}$ ). Another finding of the physical examination was wrist sign (thumb and fifth digit overlap when around the wrist) (Figure 1). His cardiac examination revealed a grade 3/6 early diastolic murmur over the aortic area. Chest X-ray showed mild cardiomegaly and mediastinal enlargement (Figure 2). The patient underwent an echocardiogram that showed a quite dilated left ventricle, severe aortic valve insufficiency, and over-dilated aortic root involving the ascending aorta. Transthoracic echocardiography revealed a severely dilated left ventricle (left ventricular enddiastolic dimension: $9.0 \mathrm{~cm}$, left ventricular end-diastolic volume: $520 \mathrm{~mL}$ ), a giant ascending aorta measuring 11.0 $\mathrm{cm}$ and reduced systolic function (left ventricular ejection fraction: 50\%) (Figure 3). A cardiovascular computed

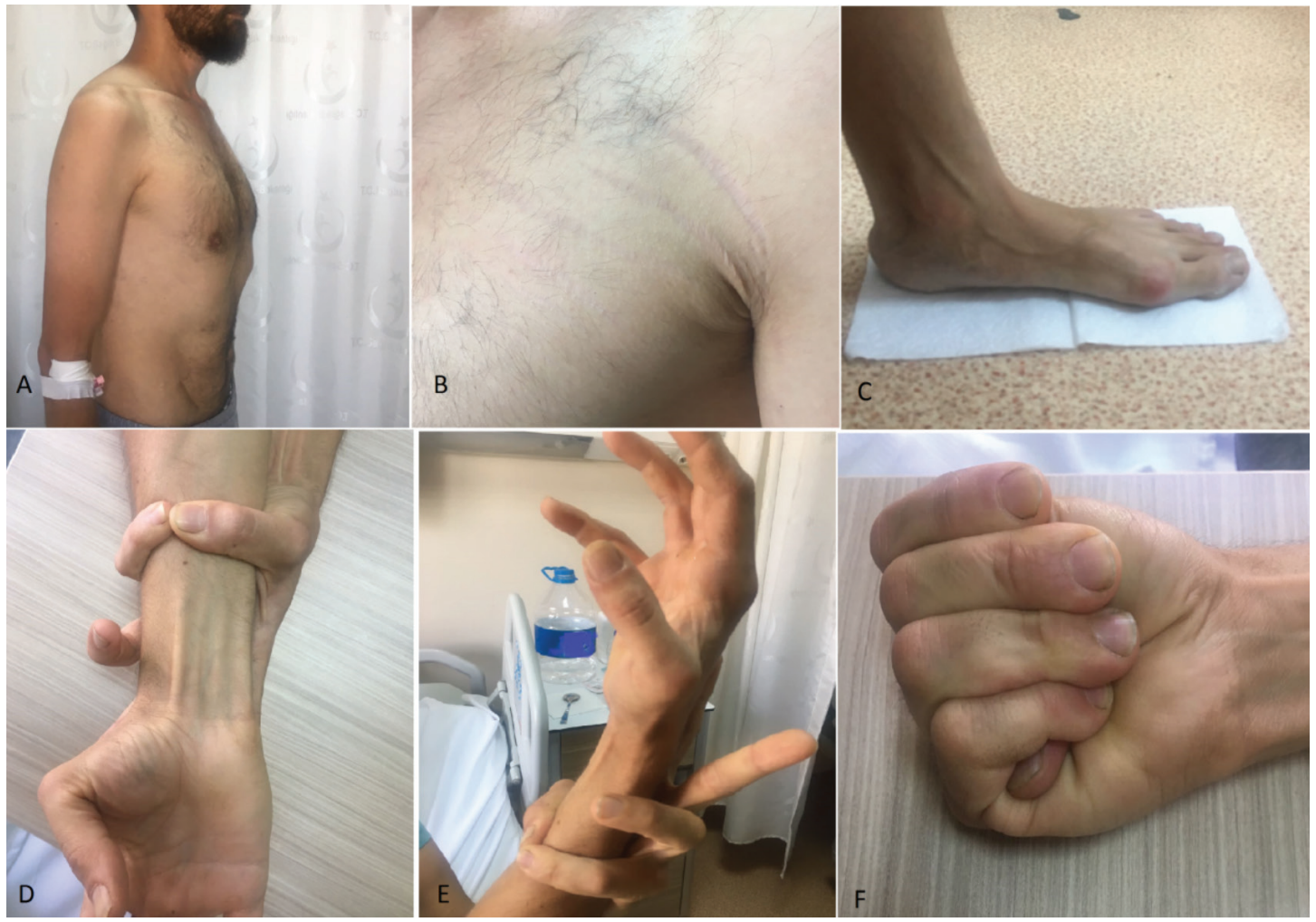

Figure 1. Skeletal characteristics of the patient: A) pectus deformity, B) striae atrophicae in axillary region, C) pes planus, D) the wrist sign, E) the wrist sign and arachnodactyly, F) the thumb sign 


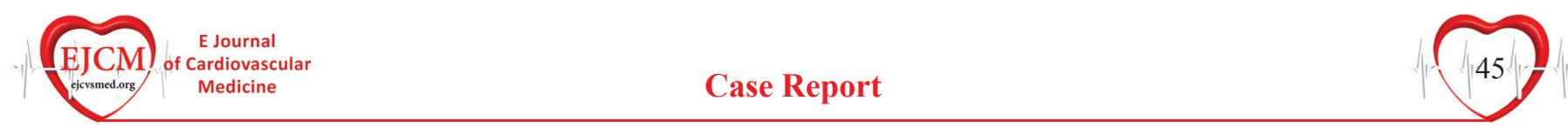

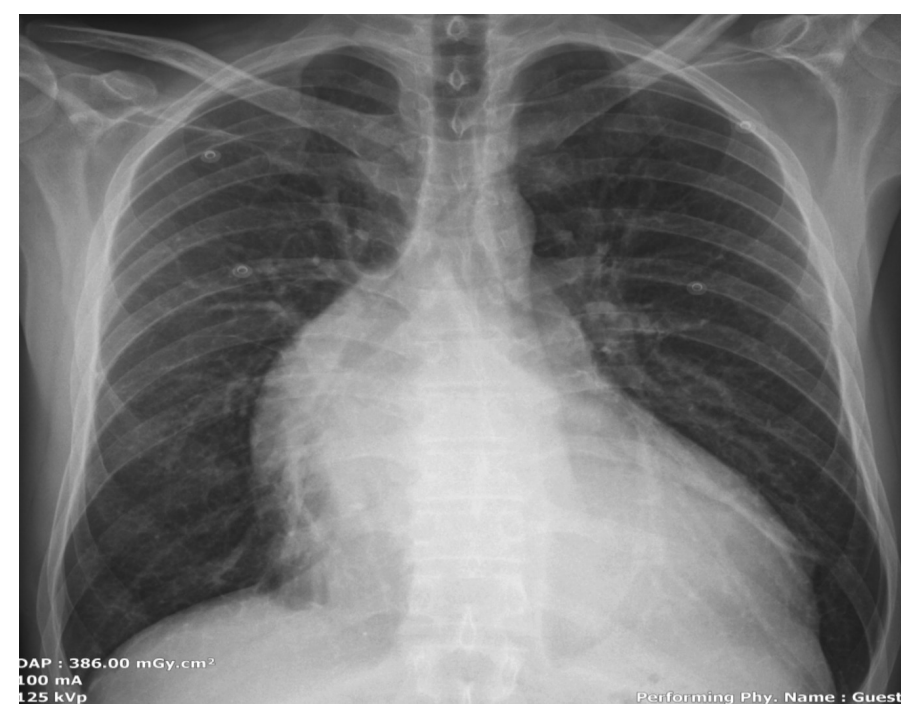

Figure 2. Chest X-ray showing cardiomegaly and mediastinal enlargement in right paracardiac area
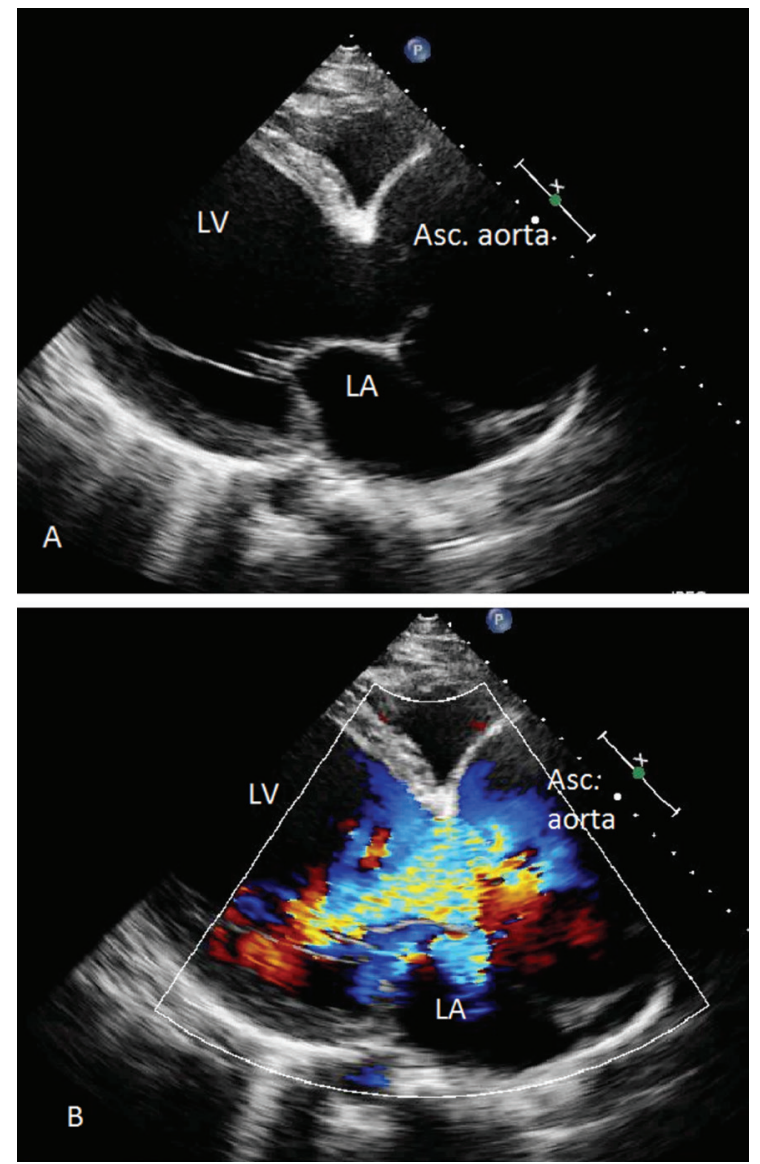

Figure 3. Transthoracic echocardiography parasternal long axis view shows, A) dilated left ventricle and ascending aortic aneurysm and, B) severe aortic regurgitation Asc.: Ascendan, LA: Left atrium, LV: Left ventricle tomography angiography (CTA) imaging study was ordered to exclude aortic dissection and assess the extent of aortic root dilation. A CTA showed an aortic diameter of $11 \times 11.6 \mathrm{~cm}$ at the widest point without any dissection flap (Figures 4 and 5). Written informed consent was obtained from the patient who underwent elective surgery.

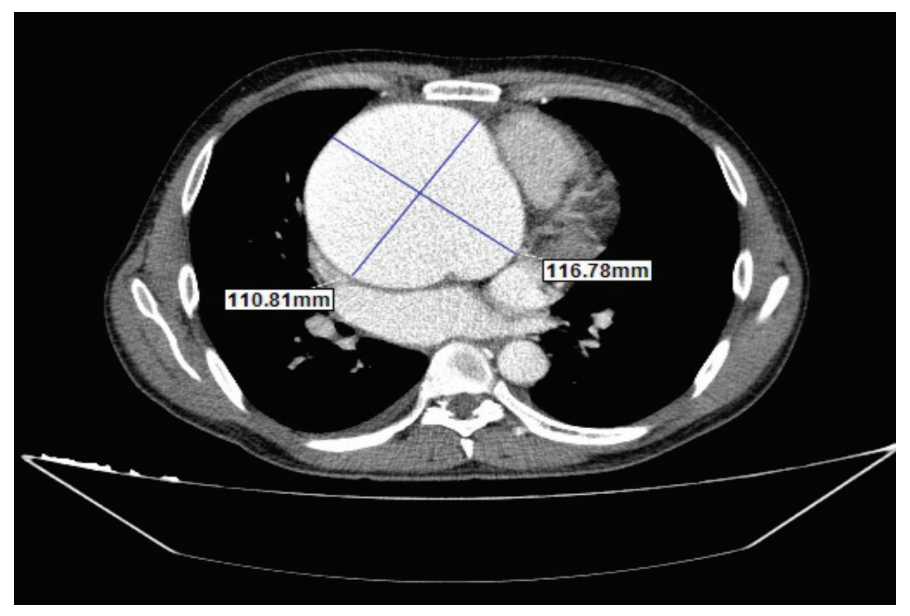

Figure 4. Computed tomography aortogram showing the dimension of ascending aorta about 110x116 mm

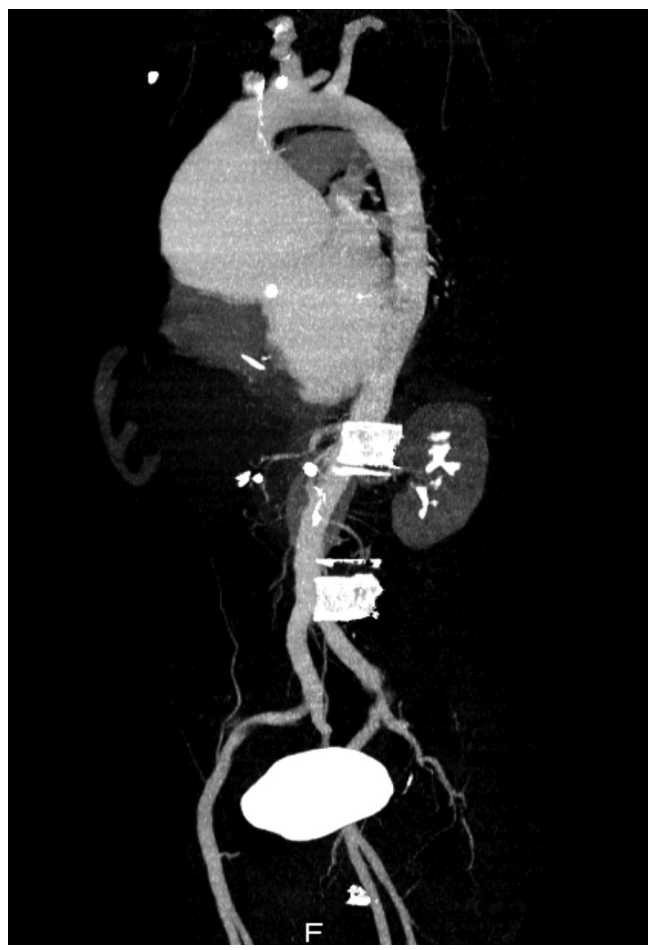

Figure 5. Computed tomography aortogram with 3D reconstruction showing fusiform dilatation involving sino-tubular junction, ascending aorta, proximal aortic arch 
The patient was diagnosed as having MFS according to the Ghent criteria and underwent Benthall procedure which involves replacement of the aortic root with a mechanical valved conduit. Following the postoperative course, he was discharged uneventfully with warfarin, metoprolol, and ramipril on the seventh day.

\section{Discussion}

Although MFS is inherited as an autosomal dominant with high penetrance, about $30 \%$ of cases are due to sporadic mutations ${ }^{(3)}$. The prevalence of MFS was reported ranging between 1/5.000-1/10.000. Cardiovascular manifestations include thoracic aortic aneurysm/dissection, aortic regurgitation from the aortic root impairment, and the mitral insufficiency with mitral valve prolapses. Although aortic dilatation is progressive throughout life, losartan and beta-blockers reduce the rate of dilatation, aortic dissection, and heart failure ${ }^{(4)}$. The incidence of ascending aortic aneurysms, defined as giant aneurysms greater than $10 \mathrm{~cm}$ in diameter, is increasing in MFS. Guidelines recommended aortic imaging of firstdegree relatives in patients with familial aortic aneurysms. The current European Society of Cardiology guidelines recommended prophylactic surgery in patients with MFS, who have a maximal aortic diameter $\geq 50 \mathrm{~mm}$. The factors that would lead to surgical repair at $\geq 45 \mathrm{~mm}$ include a family history of dissection, size increase $3 \mathrm{~mm} / \mathrm{year}$, severe aortic regurgitation, or desire for pregnancy ${ }^{(5)}$.

In the case reported, the reason that the patient became symptomatic is the presence of severe aortic valve regurgitation due to aortic root dilatation. Although the patient admitted the hospital before dissecting the aorta which is the most feared complication of MFS, the systolic function of heart was reduced and left ventricular diameters were greatly enlarged owing to severe aortic regurgitation. Despite a negative family history, the current-reported-case was sporadic. In suspicious cases, even if there is negative family history, genetic test and systemic features of MFS should be examined well and the criteria of MFS should be kept in mind. In the literature, huge aneurysm of the ascending aorta without dissection is extremely rare, and the current case with a giant aneurysm of approximately $12 \mathrm{~cm}$ has been treated uneventfully.

In conclusion, such huge aortic aneurysms could be asymptomatic and silent for many years until severe aortic regurgitation and aortic dissection occurs.

\section{Ethics}

Informed Consent: Written informed consent was obtained from the patient who underwent elective surgery.

Peer-review: Internally peer-reviewed.

\section{Authorship Contributions}

Surgical and Medical Practices: M.T., Concept: E.Ç.Ş., Ö.Ö., Design: E.Ç.Ş., Ö.Ö., Data Collection or Processing: E.Ç.Ş., E.Ö.U., M.T., Analysis or Interpretation: E.Ç.Ş., Ö.Ö., Literature Search: E.Ç.Ş., E.Ö.U., Writing: E.Ç.Ş., E.Ö.U.

Conflict of Interest: No conflict of interest was declared by the authors.

Financial Disclosure: The authors declared that this study received no financial support.

\section{References}

1. Ramirez F, Dietz HC. Marfan syndrome: from molecular pathogenesis to clinical treatment. Curr Opin Genet Dev. 2007;17:252-8.

2. Loeys BL, Dietz HC, Braverman AC, et al.The revised Ghent nosology for the Marfan syndrome. J Med Genet. 2010;47:476-85.

3. Toudjarska I, Kilpatrick MW, Lembessis P, et al. Novel approach to the molecular diagnosis of Marfan syndrome: application to sporadic cases and in prenatal diagnosis. Am J Med Genet. 2001;99:294-302.

4. Stuart AG, Williams A. Marfan's syndrome and the heart. Arch Dis Child 2007;92:351-6.

5. Erbel R, Aboyans V, Boileau C, Bossone E, Bartolomeo RD, Eggebrecht H, et al. 2014 ESC Guidelines on the diagnosis and treatment of aortic diseases: Document covering acute and chronic aortic diseases of the thoracic and abdominal aorta of the adult. The Task Force for the Diagnosis and Treatment of Aortic Diseases of the European Society of Cardiology (ESC). Eur Heart J. 2014;35:2873-926. 